\title{
Complication Rates Using CASPER Dual-Layer Stents for Carotid Artery Stenting in Acute Stroke
}

\author{
A 3-Year Single Center Experience
}

F. Runck ${ }^{1,2}(\mathbb{D})$ C. J. Maurer ${ }^{1}(\mathbb{D})$ A. Berlis ${ }^{1}(\mathbb{D})$

\begin{abstract}
Background and Purpose The number of acute and early stent occlusions after emergency stenting of the internal carotid artery (ICA) in patients with tandem lesions is unclear and only mentioned in a small number of publications, ranging from $0-20 \%$. A recent article by Yilmaz et al. reported a high rate of acute in-stent occlusions of $45 \%$ within $72 \mathrm{~h}$ after deployment of CASPER dual layer stents.

Methods All patients with acute ischemic stroke treated with a CASPER stent between August 2014 and April 2018 were retrospectively evaluated for occlusion rates, periinterventional medication and early complications. A total of 66 patients, 45 with tandem pathologies and 21 with proximal ICA stenosis only were enrolled.

Results Thrombotic complications occurred in 16 out of 66 patients (24\%) and hemorrhagic complications in 8/66 (12\%) and 15 of the 16 thrombotic complications and 7/8 symptomatic intracranial hemorrhages (sICH) occurred in patients with tandem lesions and accessory intracranial thrombectomy. Of the patients with $\mathrm{sICH}$ five were treated in a prolonged or unknown time window. In patients with intraprocedural thrombotic complications ultrasound imaging showed patent stents in 13 of the 14 patients

Conclusions The CASPER stent system showed a high technical success rate in patients with acute stroke. The number of patients with sICH was not higher than the numbers reported in the published literature despite the early use of Gp IIb/IIIA inhibitors, whereas the number of thrombotic complications was smaller than that reported in recent studies. The majority of sICH occurred in patients treated because of tandem lesions in an unknown or prolonged time window.
\end{abstract}

\author{
Abbreviations \\ +ic Carotid stent and intracranial thrombectomy \\ -ic Carotid stent only \\ ASA Acetylsalicylic acid \\ CCDS Color coded duplex sonography \\ CTA Computed tomograph angiography \\ Gp IIb/IIIa Glycoprotein IIb/IIIa \\ F. Runck \\ frank.runck@bkh-guenzburg.de \\ 1 Department of Diagnostic and Interventional Radiology and \\ Neuroradiology, Universitätsklinikum Augsburg, Augsburg, \\ Germany \\ 2 Department of Neuroradiology, \\ Bezirkskrankenhaus Günzburg, \\ Ludwig-Heilmeyer-Str. 2, 89312 Günzburg, Bavaria, Germany
}

mTICI Modified treatment in cerebral ischemia scale NCCT Non-contrast cranial computed tomography PTA Percutaneous balloon angioplasty rtPA recombinant tissue plasminogen activator sICH Symptomatic intracranial hemorrhage

\section{Introduction}

In a recent meta-analysis of five large thrombectomy trials [1] approximately $10 \%$ of patients presented with tandem lesions defined as a combination of high-grade stenosis or occlusion of the ipsilateral proximal internal carotid artery (ICA) and an occlusion of large intracranial arteries in the anterior circulation. Although this subgroup showed substantial benefits by mechanical thrombectomy, the optimal 
approach regarding the treatment of a proximal ICA lesion alone [2-4] or in combination with an intracranial occlusion [5-16] in patients with acute ischemic stroke remains unclear. A recent retrospective analysis of 295 patients showed a favorable outcome in patients with emergency stenting compared to conservative management or percutaneous balloon angioplasty (PTA) of the ICA lesion alone [17]. Aside from the optimal approach considering treatment of the proximal carotid lesions, questions about periprocedural interventional antithrombotic treatment [7, 18-20] as well as the optimal choice of stents have been raised. Although carotid stents have been available for some years, novel dual layer closed cell stents like the CASPER ${ }^{\mathrm{TM}}$ stent (Terumo Microvention, Aliso Viejo, CA, USA) or the CGuard stent (InspireMD, Boston, MA, USA) promise better plaque coverage due to smaller cell size compared to the older generation stents, thus minimizing the risk of thromboembolic events during placement and show promising results in elective patients [21-24]. The issue of early stent occlusion within 24-72 h after stenting of the ICA in patients with tandem pathologies has only been addressed in a small number of studies with numbers ranging between $0 \%$ and 19\% [8, $9,15,16,25-29]$. A recent article by Yilmaz et al. reported a surprisingly high rate of acute in-stent occlusions within $72 \mathrm{~h}$ after deployment in $45 \%$ of the patients treated with the CASPER stent in cases with tandem lesions [30]. These findings are not in line with our own experience. Therefore, this retrospective study was conducted including all emergency patients treated in the Augsburg University hospital between August 2014 and April 2018 with a CASPER stent to evaluate the incidence of thrombotic and hemorrhagic complications in these subsets of patients.

\section{Methods}

This retrospective study was approved by the local ethics committee. Because of the retrospective design informed consent was not necessary according to the committee's decision. During the study period 204 patients received a carotid stent. Of these, 164 patients were treated with a CASPER stent. Most of the other patients received either carotid wall stents as the previously used standard stent or the CGuard, which was tested during this period. Of the patients 74 receiving a CASPER stent were treated for an acute stroke, 8 patients were excluded, 2 because of failure of recanalization of intracranial thromboembolic ICA occlusions and 6 because of arterial intracranial bleeding as a complication of intracranial thrombectomy requiring occlusion of the bleeding vessels. For these patients no sufficient follow-up data were available. A total of 66 patients were included in the final analysis. Patients were divided into a group with tandem lesions receiving stenting and thrombectomy (+ic) and patients who were treated only with stenting (-ic). Postprocedural imaging as well as clinical reports were evaluated for:

1. Periinterventional medication

2. In-stent thrombosis or occlusion during intervention or hospital stay

3. Periinterventional medication, in-stent thrombosis or occlusion during intervention or hospital stay and symptomatic intracranial hemorrhage (parenchymal hemorrhage $\mathrm{PH} 1$ and 2 according to the European carotid acute stroke study (ECASS) criteria [31]).

\section{Imaging}

All emergency patients initially received a standard imaging protocol including a non contrast cranial computed tomography (NCCT) followed by a computed tomography angiography (CTA). In case of unknown onset of symptoms, e.g. wake up stroke, an additional CT perfusion (CTP) was performed. Patients were treated if a significant mismatch between cerebral blood flow (CBF) and cerebral blood volume (CBV) of more than one third of the territory of the middle cerebral artery or in eloquent regions, e.g. motor cortex, was detected.

\section{Endovascular Procedure}

Digital subtraction angiography (DSA) was usually performed at the institution neuroradiology suite on a biplane Siemens Axiom Artis (Siemens Healthcare, Erlangen, Germany). The choice of the guiding catheter and, in the case of tandem lesions, the method of thrombectomy (e.g. aspiration alone, stent retrieving or combination) as well as the choice of thrombectomy device remained at the discretion of the interventionalist. In most cases of tandem lesions an antegrade approach with primary stenting of the ICA followed by intracranial thrombectomy was chosen. Percutaneous balloon angioplasty (PTA) before stenting was only performed if the stenosis or occlusion could not primarily be passed with the stent delivery system. In cases of residual stenosis, poststenting angioplasty was performed. Usually for prestenting a $2.0 \times 20 \mathrm{~mm}$ and for poststenting a $5.0 \times 20 \mathrm{~mm}$ noncompliant balloon were used.

\section{Choice of Antiplatelet Medication}

All eligible emergency patients recombinant tissue plasminogen activator (rtPA) adapted to body weight. If no antiplatelet therapy was given before the intervention for other reasons, the institutional standard medication consisted of an i.v. dose of $100 \mathrm{mg}$ acetylsalicylic acid (ASA) after stent deployment and postprocedural administration of tirofiban. 
A $250 \mathrm{ml}$ infusion containing $12.5 \mathrm{mg}$ of tirofiban was administered i.v. with a bolus over three minutes and subsequent maintenance doses each adapted to the patient's body weight, ranging from a bolus of $25 \mathrm{ml}$ and maintenance dose of $9 \mathrm{ml} / \mathrm{h}$ for a body weight of $50 \mathrm{~kg}$ to $60 \mathrm{ml}$ and $22 \mathrm{ml} / \mathrm{h}$ for $120 \mathrm{~kg}$. At a time $4 \mathrm{~h}$ before cessation of the tirofiban infusion, a loading dose of $180 \mathrm{mg}$ of ticagrelor was administered orally, followed by a maintenance dose of $90 \mathrm{mg}$ given twice a day for a minimum of 6 weeks and $100 \mathrm{mg}$ of ASA given orally daily for at least 6 months. Administration of heparin, usually 5000IU i. v., during stent deployment was at the discretion of the interventionalist. In cases of sufficient ICA flow restoration, i.e. no or little residual stenosis according to North American Symptomatic Carotid Endarterectomy Trial (NASCET) criteria [32], some patients received only ASA, and ticagrelor was administered after the $24 \mathrm{~h}$ NCCT. No protection devices were used. Most treatments were carried out with the patients under general anesthesia.

\section{Postinterventional Imaging}

In cases of intracranial thrombectomy, a flat detector NCCT (DynaCT ${ }^{\mathrm{TM}}$, Siemens, Erlangen, Germany) was routinely performed after the procedure. If no intracranial thrombectomy was performed, patients did not undergo immediate postprocedural computed tomography (CT) imaging. All patients routinely received a postprocedural NCCT $24 \mathrm{~h}$ after treatment. In cases of neurologic deterioration or $\mathrm{sICH}$ on flat panel NCCT, additional postprocedural NCCT was performed.

Postprocedural CCDS examinations were available for $35(78 \%)+$ ic patients and $20(95 \%)$-ic patients (Table 1$)$. In only one patient who received a stent due to a complication of a surgical procedure, the first ultrasound examination was performed only transcranially, only enabling evaluation of indirect signs of proximal ICA pathology. Ultrasound examinations were performed by the neurologist on duty.
Table 1 Demographic data, preprocedural and postprocedural stenosis grading

Table 2 Periprocedural medication

\begin{tabular}{lll}
\hline & Stent and thrombectomy $(n=45)$ & Stent only $(n=21)$ \\
\hline Age (years) & $65 \pm 10$ & $74 \pm 9$ \\
Sex female & $13(29 \%)$ & $6(29 \%)$ \\
Side left & $27(60 \%)$ & $11(52 \%)$ \\
Diabetes & $9(20 \%)$ & $9(43 \%)$ \\
Hypertension & $34(76 \%)$ & $20(95 \%)$ \\
Coronary artery disease & $6(13 \%)$ & $8(38 \%)$ \\
Dyslipidemia & $27(60 \%)$ & $16(76 \%)$ \\
Previous stroke & $10(22 \%)$ & $9(43 \%)$ \\
Stenosis DSA preprocedural $(\%)$ & $93 \pm 16$ & $90 \pm 9$ \\
Stenosis DSA postprocedural $(\%)$ & $11 \pm 22$ & $9 \pm 12$ \\
Stenosis CCDS postprocedural $(\%)$ & $12 \pm 28(n=35)$ & $11 \pm 189(n=20)$ \\
\hline
\end{tabular}

$D S A$ digital subtraction angiography, $C C D S$ color coded duplex sonography

\begin{tabular}{lll}
\hline Medication & Stent and thrombectomy $(n=45)$ & Stent only $(n=21)$ \\
\hline rtPA (\%) & $33(73 \%)$ & $9(43 \%)$ \\
ASA preprocedural & $12(27 \%)$ & $12(57 \%)$ \\
Clopidogrel preprocedural & $2(4 \%)$ & $3(14 \%)$ \\
Ticagrelor preprocedural $(\%)$ & 0 & 0 \\
Mono-platelet inhibition preprocedural & $12(27 \%)$ & $13(62 \%)$ \\
Dual platelet inhibition preprocedural & $1(2 \%)$ & $1(5 \%)$ \\
ASA intraprocedural & $39(87 \%)$ & $9(43 \%)$ \\
Tirofiban intraprocedural & $33(73 \%)$ & $9(43 \%)$ \\
Heparin intraprocedural & $9(20 \%)$ & $6(29 \%)$ \\
ASA postprocedural $(\%)$ & $41(91 \%)$ & $20(95 \%)$ \\
Clopidogrel postprocedural $(\%)$ & $6(13 \%)$ & $4(19 \%)$ \\
Ticagrelor postprocedural $(\%)$ & $34(76 \%)$ & $15(71 \%)$ \\
\hline
\end{tabular}

$r t P A$ recombinant tissue plasminogen activator, $A S A$ acetylsalicylic acid 


\section{Results}

Details concerning the demographic data are given in Table 1 and the medication in Table 2 . The only obvious differences between the two groups were the higher mean age $(74 \pm 9.4$ years vs. $64 \pm 10.1$ years $)$ and the higher number of patients receiving ASA (57\% vs 27\%) in the stent only group. Not listed in the tables are ASPECTS on admission- (+ic 8.0 \pm 2.2 , -ic 9.5 \pm 1.0$)$ and on follow-up NCCT (+ic $6.8 \pm 1.3$, -ic $8.0 \pm 1.8$ ) as well as National Institutes of Health Stroke Scale (NIHSS) scores on admission (+ic $12.9 \pm 5.3$, -ic 8.1 \pm 3.9$)$ and at discharge (+ic 6.7 \pm 5.2 , -ic 5.4 \pm 4.4$)$. The NIHSS scores were not available for all of the patients.

An antegrade approach with initial stenting of the ICA followed by intracranial thrombectomy was undertaken in $36(80 \%)$ and a retrograde approach in $9(20 \%)$ patients. Predilation was necessary in 4 patients (+ic $3 / 7 \%$, -ic $1 / 5 \%$ ), postdilation in 33 (+ic 23/51\%, -ic 10/48\%) and both in 16 patients (+ic $11 / 24 \%$, -ic $7 / 33 \%$ ). In thrombectomy patients the level of intracranial occlusion was distal ICA or carotid-T in 13, M1 in 23, and M2 in 9 patients (29\%, $51 \%$, and $20 \%$, respectively). Final recanalization results according to the modified treatment in cerebral ischemia scale (mTICI) [33] were 0 in 2 (4\%), 2a in $2(4 \%), 2 b$ in $27(60 \%)$ and 3 in $14(31 \%)$ patients.

Thrombotic complications occurred in 16/66 (24\%) patients and hemorrhagic complications in 8/66 (12\%). Most of these complications (15/16 thrombotic, $7 / 8 \mathrm{sICH})$ occurred in patients with tandem lesions, corresponding to a rate of $33 \%$ thrombotic and $16 \%$ sICH in this subset of 45 patients and 1 patient suffered both complications. Of the patients 2 showed delayed sICH more than $24 \mathrm{~h}$ after intervention, 2 patients, who are not listed, showed mild subarachnoid hemorrhage on $24 \mathrm{~h}$ follow-up NCCT but were asymptomatic. Of the patients with $\mathrm{sICH}, 2$ patients had a wake-up stroke and 3 patients were treated in an extended time window more than $6 \mathrm{~h}$ after onset.

Intraprocedural thrombotic complications were usually detected at the end of the procedure during cervical control angiography. Of the 14 patients with intraprocedural thrombotic complications 3 showed an intraprocedural complete stent occlusion and 11 thrombotic stent wall apposition resulting in a significant stenosis of at least $50 \%$. In cases of complete occlusion treatment consisted in one case of aspiration thrombectomy and administration of tirofiban, in the second case of administration of papaverine for spasmolysis and tirofiban and in the third case of tirofiban only. Of these patients one developed sICH and died and the two remaining patients showed patent stents without significant stenosis on follow-up CCDS. Overall 11 patients, 10 with tandem lesions and 1 with stenting alone, showed intraprocedural thrombotic apposition at the stent wall. Of these,
Table 3 Complications by patient groups

\begin{tabular}{lll}
\hline & $\begin{array}{l}\text { Stent and thrombectomy } \\
(n=45)\end{array}$ & $\begin{array}{l}\text { Stent only } \\
(n=21)\end{array}$ \\
\hline sICH & $7(16 \%)$ & $1(5 \%)$ \\
Intraprocedural & $3(7 \%)$ & - \\
occlusion & & - \\
Early occlusion & $2(4 \%)$ & $1(4 \%)$ \\
In-stent thrombosis & $10(22 \%)$ & \\
\hline
\end{tabular}

sICH symptomatic intracranial hemorrhage

one patient was treated with PTA and accessory stent and three with PTA only. All other patients received tirofiban as sole treatment. All of these patients received dual antiplatelet therapy overlapping with the tirofiban infusion.

Early occlusion occurred in two cases, in one case $6 \mathrm{~h}$ after intervention and during tirofiban infusion. The second patient, who had a thrombotic stent wall apposition during procedure, showed progression to a complete stent occlusion on CCDS examination the day following the intervention, most likely because of early cessation of antiplatelet therapy due to gastrointestinal bleeding.

For 14 of the 16 patients with thrombotic complications CCDS follow-up was available, showing only 1 occlusion and in 2 patients a stenosis of $60 \%$. An overview on the number of complications for each group is provided in Table 3.

\section{Discussion}

The benefits of endovascular treatment of symptomatic as well as asymptomatic ICA stenosis was shown in a recent follow-up analysis of the CREST study [34]. There are only a few smaller retrospective studies that evaluated the benefits of emergency endovascular treatment of isolated symptomatic ICA stenosis or occlusion. These reported high recanalization rates and good clinical outcomes [2-4]. The HERMES meta-analysis of five large thrombectomy studies additionally showed beneficial effect of endovascular ICA treatment in patients with tandem lesions [1]. Furthermore, a number of meta-analyses and retrospective case series documented the safety and technical feasibility of treatment of proximal ICA pathology in patients with tandem lesions, mostly with PTA-assisted stenting [7-14, 17]. Due to the lack of prospective data, the optimal treatment of proximal ICA pathology in patients presenting with acute stroke and especially with tandem lesions, remains the subject of discussion mainly because of concerns that the necessary platelet inhibition in combination with acute brain infarction might lead to a higher rate of $\operatorname{sICH}[5,25,35]$.

Only a few articles addressing the treatment of tandem pathologies mentioned the number of early stent occlusions with numbers ranging from $0 \%$ to $19 \%[8,9,15,16,25-29]$. 
In these articles mostly monolayer stents were used and the periprocedural antithrombotic medication differed significantly between the studies especially with respect to the use of $\mathrm{Gp}$ IIb/IIIa inhibitors. Compared to these numbers a recent study by Yilmaz et al. [30] showed a surprisingly high rate of in-stent thrombosis or stent occlusion in patients with acute tandem lesions treated with CASPER dual layer stents of $50 \%$ and $45 \%$, respectively compared to monolayer stents ( $37 \%$ and $3.7 \%$, respectively), thus raising the question of the optimal stents to be used in these cases. On the other hand recent reports indicated promising results in the treatment of elective patients with dual layer stents, mostly in combination with distal protection devices, in terms of technical success [23] and reduction of thrombus protrusion through the stent cells as well as a reduction in postinterventional diffusion weighted imaging lesions on magnetic resonance imaging [22, 24], most likely due to smaller cell size compared to older generation monolayer stent systems. Because of its mechanical properties the CASPER stent seems to be well-suited for use in stroke patients [23, 24].

In most of the cases in this study with significant intraprocedural thrombus formation, the most likely cause was a wedge position of either the aspiration catheter or the access catheter which resulted in blood stasis within the stent lumen and significant in-stent thrombosis of more than $50 \%$. Of the patients with significant in-stent thrombus one showed severe vasospasm after stent deployment and stasis within the vessel lumen as the most probable mechanism leading to the thrombotic formation at the stent wall.

The percentage of patients with acute and early occlusions of $11 \%$ is in line with the numbers reported for the monolayer stents mentioned above and much lower than reported in the recent report by Yilmaz et al. [30]. In this emergency cohort, on the other hand a relatively high number of in-stent thrombus appositions was also found in the -+ic group, although the numbers were far lower than in the work of Yilmaz et al. (+ic 33\% vs. 56\%) [30]. The suggested mechanism is a higher thrombogenicity of the CASPER stent because of the smaller cell size resulting in a higher metal-artery ratio. Another reason for the higher rate of in-stent thrombosis in the $+\mathrm{ic}$ group might be the delayed restoration of adequate blood flow in patients with a concomitant intracranial lesion when treating the extracranial lesion first. This might be especially true in patients with occlusion of the carotid $\mathrm{T}$.

The most likely explanation of the much lower incidence of early occlusion in this series is a more aggressive antiplatelet regimen. Compared to the population of patients reported by Yilmaz et al. [30] the number of acute patients with single or dual antiplatelet treatment before stenting was slightly (+ic $29 \%$, -ic $67 \%$, overall $41 \%$ vs $52 \%$ ), while the percentage of patients receiving i.v. thrombolysis was higher in the present cohort (+ic $73 \%$, -ic $43 \%$, overall $64 \%$ vs. 30\%). The general antiplatelet regimen used by Yilmaz et al. [30] in patients without preinterventional antiplatelet medication consisted of $500 \mathrm{mg}$ of ASA i.v. during stent deployment and clopidogrel within $24 \mathrm{~h}$ postintervention, starting with a loading dose of $300 \mathrm{mg}$. In contrast to this relatively conservative approach by default tirofiban was already used during interventions in most cases and dual platelet inhibition was started usually as a combination of ASA and ticagrelor before the end of the tirofiban administration. Furthermore, Yilmaz et al. observed a thrombus formation or thrombus protrusion in $37 \%$ of the patients treated with monolayer stents and in $50 \%$ of the patients treated with dual layer stents, progressing to complete occlusion in $55 \%$ of the patients. The use of clopidogrel did not seem to influence the rate of stent occlusions [30]. Although the rate of significant in-stent thrombosis of $33 \%$ in patients with tandem lesions seems relatively high, progression to a stent occlusion was observed in only one of these patients. In this patient, however, no sufficient platelet inhibition could be administered because of gastrointestinal bleeding. These findings are similar to the results of Steglich-Arnholm et al. [15], who observed acute stent thrombosis during endovascular procedure when mainly monolayer stents were used in 8 out of $47(17 \%)$ of the patients. In 7 of these, administration of $\mathrm{Gp}$ IIb/IIIa antagonists (eptifibatide or abciximab) led to recanalization. Despite the much more aggressive antiplatelet therapy in the present cohort of emergency patients, the rate of $\mathrm{sICH}$ was $12 \%(+\mathrm{ic} 16 \%$, -ic $5 \%)$ and therefore within the numbers reported in the literature ranging from $4 \%$ to $20 \%[6,7,9,11,15,25,30]$. Remarkably, all but one sICH occurred in the group with tandem lesions. Of these patients two were treated in an extended time window with known onset but no or little infarct demarcation and three as a wake-up stroke. Furthermore, although not available for all patients, the lower average ASPECTS and higher NIHSS scores in the +ic-group might be suggestive of a more severe ischemia and might partly explain the higher incidence of intracranial hemorrhage in this group.

Delayed administration of clopidogrel and abdication of $\mathrm{Gp}$ IIb/IIIa inhibitors did not lead to a reduced number of sICH (5\% dual layer, $18.5 \%$ mono layer, overall 22\%) in the cohort of Yilmaz et al. [30]. The high rate of thrombotic complications in this cohort might be due to the reported high rate of clopidogrel nonresponders of up to $45 \%$ [36]. Intravenous tirofiban immediately after rtPA seems to be safe and potentially more effective when compared to alteplase alone for selected stroke patients and not leading to a significantly higher rate of ICH [37]. On the other hand, a recent meta-analysis reported only a slightly increased risk of $\mathrm{sICH}$ in patients with acute stroke receiving antiplatelet medication [38]. This risk has to be weighed against the complications of an ICA reocclusion, since no 
prospective data exist on the problem of medication after stent implantation in acute stroke, therefore, no clear recommendations for antiplatelet therapy after CAS can be given in these cases [19, 39], although many authors advocate early use of Gp IIb/IIIa receptor inhibitors [7, 18-20].

This study has some obvious limitations. First, it is a monocentric and retrospective cohort study. Second, antiplatelet medication varied between patient groups as well as within the groups despite a fixed institutional medication regimen, which makes it difficult to determine the influence of the antiplatelet therapy on the occurrence of adverse events. In addition, no conclusions can be drawn on the influence of thrombotic complications on the clinical outcome since the modified Rankin scale was not routinely assessed after 90 days. The influence of periprocedural and postprocedural complications on clinical outcome therefore remains unclear in this cohort.

\section{Conclusion}

In this series of CASPER stents used in the treatment of acute ischemic stroke, the number of patients with symptomatic intracranial hemorrhage with early administration of Gp IIb/IIIA inhibitors and dual platelet inhibition was not higher than the numbers reported in the literature. The number of thrombotic complications was well below that reported in recent studies evaluating CASPER stents in patients with tandem lesions. Given the high numbers of patients with sICH who were in an unclear or prolonged time window due to tandem lesions, stent treatment in these patient groups should only be performed after a thorough riskbenefit analysis. The use of the CASPER dual layer stent in acute stroke patients showed an acceptably low complication rate. Further prospective studies are needed to address the question of optimal treatment strategies as well as antiplatelet therapy in patients with emergency stenting with or without tandem lesions.

\section{Compliance with ethical guidelines}

Conflict of interest F. Runck declares that he has no competing interests. C.J. Maurer received educational grants from Microvention and Stryker. A. Berlis is proctor for Sequent Medical, Microvention, Stryker and Medtronic and received lecture royalties from Penumbra.

Ethical standards This article does not contain any studies with human participants or animals performed by any of the authors. All investigations described in this manuscript were carried out with the approval of the responsible ethics committee and in accordance with national law and the Helsinki Declaration of 1975 (in its current revised form).

\section{References}

1. Goyal M, Menon BK, van Zwam WH, Dippel DW, Mitchell PJ, Demchuk AM, et al. Endovascular thrombectomy after large-vessel ischaemic stroke: a meta-analysis of individual patient data from five randomised trials. Lancet. 2016;387:1723-31.

2. Jovin TG, Gupta R, Uchino K, Jungreis CA, Wechsler LR, Hammer $\mathrm{MD}$, et al. Emergent stenting of extracranial internal carotid artery occlusion in acute stroke has a high revascularization rate. Stroke. 2005;36:2426-30.

3. Son S, Choi DS, Oh MK, Kim SK, Kang H, Park KJ, et al. Emergency carotid artery stenting in patients with acute ischemic stroke due to occlusion or stenosis of the proximal internal carotid artery: a single-center experience. J Neurointerv Surg. 2015;7:238-44.

4. Nakagawa N, Fukawa N, Tsuji K, Nakano N, Murakami S, Nagatsuka $\mathrm{K}$, et al. Urgent carotid artery stenting for carotid-related stroke-in-evolution. Oper Neurosurg (Hagerstown). 2018;14:9-15.

5. Dorado L, Castaño C, Millán M, Aleu A, de la Ossa NP, Gomis $\mathrm{M}$, et al. Hemorrhagic risk of emergent endovascular treatment plus stenting in patients with acute ischemic stroke. J Stroke Cerebrovasc Dis. 2013;22:1326-31.

6. Stampfl S, Ringleb PA, Möhlenbruch M, Hametner C, Herweh C, Pham M, et al. Emergency cervical internal carotid artery stenting in combination with intracranial thrombectomy in acute stroke. AJNR Am J Neuroradiol. 2014;35:741-6.

7. Behme D, Mpotsaris A, Zeyen P, Psychogios MN, Kowoll A, Maurer CJ, et al. Emergency stenting of the extracranial internal carotid artery in combination with anterior circulation thrombectomy in acute Ischemic stroke: a retrospective multicenter study. AJNR Am J Neuroradiol. 2015;36:2340-5.

8. Cohen JE, Gomori JM, Rajz G, Itshayek E, Eichel R, Leker RR. Extracranial carotid artery stenting followed by intracranial stentbased thrombectomy for acute tandem occlusive disease. J Neurointerv Surg. 2015;7:412-7.

9. Lockau H, Liebig T, Henning T, Neuschmelting V, Stetefeld H, Kabbasch C, et al. Mechanical thrombectomy in tandem occlusion: procedural considerations and clinical results. Neuroradiology. 2015;57:589-98.

10. Kappelhof M, Marquering HA, Berkhemer OA, Majoie CB. Intraarterial treatment of patients with acute ischemic stroke and internal carotid artery occlusion: a literature review. J Neurointerv Surg. 2015;7:8-15.

11. Maurer CJ, Joachimski F, Berlis A. Two in one: endovascular treatment of acute tandem occlusions in the anterior circulation. Clin Neuroradiol. 2015;25:397-402.

12. Marnat G, Mourand I, Eker O, Machi P, Arquizan C, Riquelme C, et al. Endovascular management of tandem occlusion stroke related to internal carotid artery dissection using a distal to proximal approach: insight from the RECOST study. AJNR Am J Neuroradiol. 2016;37:1281-8.

13. Sivan-Hoffmann R, Gory B, Armoiry X, Goyal M, Riva R, Labeyrie $\mathrm{PE}$, et al. Stent-retriever thrombectomy for acute anterior ischemic stroke with tandem occlusion: a systematic review and meta-analysis. Eur Radiol. 2017;27:247-54.

14. Mbabuike N, Gassie K, Brown B, Miller DA, Tawk RG. Revascularization of tandem occlusions in acute ischemic stroke: review of the literature and illustrative case. Neurosurg Focus. 2017;42:E15.

15. Steglich-Arnholm H, Holtmannspötter M, Kondziella D, Wagner A, Stavngaard T, Cronqvist ME, et al. Thrombectomy assisted by carotid stenting in acute ischemic stroke management: benefits and harms. J Neurol. 2015;262:2668-75.

16. Mpotsaris A, Kabbasch C, Borggrefe J, Gontu V, Soderman M. Stenting of the cervical internal carotid artery in acute stroke management: the Karolinska experience. Interv Neuroradiol. 2017;23:159-65. 
17. Zhu F, Bracard S, Anxionnat R, Derelle AL, Tonnelet R, Liao L, et al. Impact of emergent cervical carotid Stenting in tandem occlusion strokes treated by thrombectomy: a review of the TITAN collaboration. Front Neurol. 2019;10:206. https://doi.org/10.3389/ fneur.2019.00206.

18. Köklü E, Yüksel İÖ, Bayar N, Arslan Ş. Is acute carotid artery stent thrombosis an avoidable complication? J Stroke Cerebrovasc Dis. 2015;24:2219-22.

19. Huibers A, Halliday A, Bulbulia R, Coppi G, de Borst GJ; ACST2 Collaborative Group. Antiplatelet therapy in carotid artery stenting and carotid endarterectomy in the asymptomatic carotid surgery trial-2. Eur J Vasc Endovasc Surg. 2016;51:336-42.

20. Moulakakis KG, Mylonas SN, Lazaris A, Tsivgoulis G, Kakisis J, Sfyroeras GS, et al. Acute carotid stent thrombosis: a comprehensive review. Vasc Endovascular Surg. 2016;50:511-21.

21. Casana R, Tolva V, Odero A Jr, Malloggi C, Paolucci A, Triulzi $\mathrm{F}$, et al. Safety and efficacy of the new micromesh-covered stent cguard in patients undergoing carotid artery stenting: early experience from a single centre. Eur J Vasc Endovasc Surg. 2017;54:681-7.

22. Musialek P, Hopkins LN, Siddiqui AH. One swallow does not a summer make but many swallows do: accumulating clinical evidence for nearly-eliminated peri-procedural and 30-day complications with mesh-covered stents transforms the carotid revascularisation field. Postepy Kardiol Interwencyjnej. 2017;13:95-106.

23. Mutzenbach SJ, Millesi K, Roesler C, Broussalis E, Pikija S, Sellner $\mathrm{J}$, et al. The casper stent system for carotid artery stenosis. J Neurointerv Surg. 2018;10:869-73.

24. Broussalis E, Griessenauer C, Mutzenbach S, Pikija S, Jansen H, Stevanovic V, et al. Reduction of cerebral DWI lesion burden after carotid artery stenting using the CASPER stent system. J Neurointerv Surg. 2019;11:62-7.

25. Heck DV, Brown MD. Carotid stenting and intracranial thrombectomy for treatment of acute stroke due to tandem occlusions with aggressive antiplatelet therapy may be associated with a high incidence of intracranial hemorrhage. J Neurointerv Surg. 2015;7:170-5.

26. Malik AM, Vora NA, Lin R, Zaidi SF, Aleu A, Jankowitz BT, et al. Endovascular treatment of tandem extracranial/intracranial anterior circulation occlusions: preliminary single-center experience. Stroke. 2011;42:1653-7.

27. Rangel-Castilla L, Rajah GB, Shakir HJ, Shallwani H, Gandhi S, Davies JM, et al. Management of acute ischemic stroke due to tandem occlusion: should endovascular recanalization of the extracranial or intracranial occlusive lesion be done first? Neurosurg Focus. 2017;42:E16.

28. Sadeh-Gonik U, Tau N, Friehmann T, Bracard S, Anxionnat R, Derelle AL, et al. Thrombectomy outcomes for acute stroke patients with anterior circulation tandem lesions: a clinical registry and an update of a systematic review with meta-analysis. Eur J Neurol. 2018;25:693-700

29. Pop R, Zinchenko I, Quenardelle V, Mihoc D, Manisor M, Richter JS, et al. Predictors and clinical impact of delayed stent thrombosis after thrombectomy for acute stroke with tandem lesions. AJNR Am J Neuroradiol. 2019;40:533-9.

30. Yilmaz U, Körner H, Mühl-Benninghaus R, Simgen A, Kraus C, Walter $\mathrm{S}$, et al. Acute occlusions of dual-layer carotid stents after endovascular emergency treatment of tandem lesions. Stroke. 2017;48:2171-5.

31. Larrue V, von Kummer R R, Müller A, Bluhmki E. Risk factors for severe hemorrhagic transformation in ischemic stroke patients treated with recombinant tissue plasminogen activator: a secondary analysis of the European-Australasian Acute Stroke Study (ECASS II). Stroke. 2001;32:438-41.

32. North American Symptomatic Carotid Endarterectomy Trial (NASCET) Steering Committee. North American Symptomatic Carotid Endarterectomy Trial. Methods, patient characteristics, and progress. Stroke. 1991;22:711-20.

33. Zaidat OO, Yoo AJ, Khatri P, Tomsick TA, von Kummer R, Saver JL, et al. Recommendations on Angiographic Revascularization Grading Standards for Acute Ischemic Stroke. Stroke. 2013;44:2650-63.

34. Brott TG, Howard G, Roubin GS, Meschia JF, Mackey A, Brooks $\mathrm{W}$, et al. Long-term results of stenting versus endarterectomy for carotid-artery stenosis. N Engl J Med. 2016;374:1021-31.

35. Coelho A, Lobo M, Gouveia R, Silveira D, Campos J, Augusto $\mathrm{R}$, et al. Overview of evidence on emergency carotid stenting in patients with acute ischemic stroke due to tandem occlusions: a systematic review and meta-analysis. J Cardiovasc Surg (Torino). 2018. https://doi.org/10.23736/S0021-9509.18.10312-0.

36. Jernberg T, Payne CD, Winters KJ, Darstein C, Brandt JT, Jakubowski $\mathrm{JA}$, et al. Prasugrel achieves greater inhibition of platelet aggregation and a lower rate of non-responders compared with clopidogrel in aspirin-treated patients with stable coronary artery disease. Eur Heart J. 2006;27:1166-73.

37. Li W, Lin L, Zhang M, Wu Y, Liu C, Li X, et al. Safety and preliminary efficacy of early tirofiban treatment after alteplase in acute ischemic stroke patients. Stroke. 2016;47:2649-51.

38. van de Graaf RA, Chalos V, Del Zoppo GJ, van der Lugt A, Dippel DWJ, Roozenbeek B. Periprocedural antithrombotic treatment during acute mechanical thrombectomy for ischemic stroke: a systematic review. Front Neurol. 2018;9:238.

39. Enomoto Y, Yoshimura S. Antiplatelet therapy for carotid artery stenting. Interv Neurol. 2013;1:151-63. 